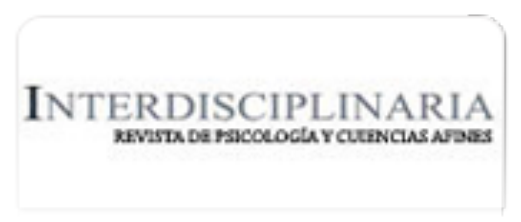

Interdisciplinaria

ISSN: 0325-8203

ISSN: 1668-7027

interdisciplinaria@fibercorp.com.ar

Centro Interamericano de Investigaciones Psicológicas y

Ciencias Afines

Argentina

Sánchez, María Elina; Jaichenco, Virginia; Sevilla, Yamila

El procesamiento del género y el número en la producción de la concordancia del español

Interdisciplinaria, vol. 35, núm. 2, 2018, Julio-, pp. 459-475

Centro Interamericano de Investigaciones Psicológicas y Ciencias Afines

Argentina

Disponible en: https://www.redalyc.org/articulo.oa?id=18058785013

Cómo citar el artículo

Número completo

Más información del artículo

Página de la revista en redalyc.org

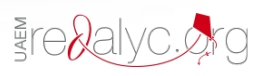

Sistema de Información Científica Redalyc

Red de Revistas Científicas de América Latina y el Caribe, España y Portugal

Proyecto académico sin fines de lucro, desarrollado bajo la iniciativa de acceso

abierto 


\title{
El procesamiento del género y el número en la producción de la concordancia del español*
}

\section{Gender and number processing in the agreement production in Spanish}

\author{
María Elina Sánchez**, Virginia Jaichenco*** y Yamila Sevilla****
}

\begin{abstract}
*Este trabajo se realizó en el marco del Proyecto UBACyT 20020110100129 "Investigaciones sobre las alteraciones léxicas y sintácticas en pacientes con patologías del lenguaje. Una ventana al sistema de procesamiento lingüístico en español" y del Proyecto PIP / CONICET 112201101 00994: "Relaciones entre procesos léxicos y procesos sintácticos en la producción y la comprensión de lenguaje".

**Doctora en Lingüística. Ayudante de la Cátedra de Neurolingüística, Facultad de Filosofía y Letras, Universidad de Buenos Aires. Becaria posdoctoral del Consejo Nacional de Investigaciones Científicas y Técnicas (CONICET). E-mail: mesanchez@filo.uba.ar

***Doctora en Lingüística. Profesora Adjunta de las Cátedras de Neurolingüística y de Psicolingüística I, Facultad de Filosofía y Letras, Universidad de Buenos Aires. Investigadora adjunta del Instituto de Lingüística, Facultad de

Filosofía y Letras, Universidad de Buenos Aires. E-mail: vjaichenco@filo.uba.ar

****Doctora en Lingüística. Jefa de trabajos prácticos de las Cátedras de Lingüística y de Neurolingüística, Facultad de Filosofía y Letras, Universidad de Buenos Aires. Investigadora adjunta del Consejo Nacional de Investigaciones Científicas y Técnicas (CONICET). E-mail: ysevilla@filo.uba.ar.

Instituto de Lingüística, Facultad de Filosofía y Letras, Universidad de Buenos Aires. Consejo Nacional de Investigaciones Científicas y Técnicas (CONICET).

Ciudad Autónoma de Buenos Aires, Argentina.
\end{abstract}

\section{Resumen}

En este trabajo se investigó el procesamiento de los rasgos de género y número en la producción de la concordancia sujeto-verbo y sujetoadjetivo predicativo del español. Bajo el paradigma de elicitación de errores diseñamos un experimento en el que se manipuló el género del sustantivo núcleo del sujeto (N1) y del sustantivo interfiriente (N2) (masculino-femenino), y el número de N1 y de N2 (singular-plural): Ejemplo: El abuelo (N1) del chico (N2). Participaron del experimento 20 sujetos a los que se les presentó en forma visual la base del adjetivo por $500 \mathrm{~ms}$ (PEINAD_) y luego el preámbulo (4000 ms.) que debían leer y completar con el verbo ser o estar y el adjetivo flexionado. Ejemplo: El abuelo del chico está peinado. A cada sujeto se le administró una lista con 128 targets. Se analizó el patrón de errores y de omisiones de la concordancia sujeto-verbo (S-V) y sujeto-pre- dicativo (S-P). Los resultados arrojaron efectos de marcación de número y de género. Los datos indicaron que los participantes produjeron más errores de concordancia S-V cuando el sustantivo interfiriente estaba en plural; mientras que el rasgo más influyente para la concordancia $\mathrm{S}$ $\mathrm{P}$ fue el género femenino del sustantivo núcleo del sujeto. Los datos obtenidos en nuestro trabajo experimental fueron analizados en términos de una teoría general del procesamiento de modo que permiten informar un modelo de producción de la concordancia aún en desarrollo.

Palabras clave: Psicolingüística; Producción de oraciones; Concordancia; Género; Número
Abstract
In this work the processing features of gen- der and number in subject-verb agreement and 
subject-predicate adjective in the sentence production in Spanish was investigated. As a general objective, in this work we set out to investigate the processing of the features that come into play in both subject-verb and subject-adjective predicative agreement in our language. Specifically, an attempt will be made to answer if the features of gender and number are processed in the same way, if the feature have a default value and if the values present differences, that is, if the singular differs from the plural, and the masculine from the feminine. This will allow us to establish if any of these values is more error-sensitive. In addition, we seek to identify the role of the marking concept. Under the paradigm of error elicitation we designed an experiment in which the Gender of the head noun (N1) and local noun (N2) (Masculine-Feminine), and the Number of N1 and N2 (Singular-Plural) was manipulated: Example: El abuelo (N1) del niño (N2). 20 subjects participated in the experiment which were presented in visual form the basis of the adjective for $500 \mathrm{~ms}$. (PEINAD_) and then the preamble $(4000 \mathrm{~ms}$.) The participants read and completed with the verb "to be" and inflected adjective: Example: El abuelo del niño está peinado. Each participant was given a list of 128 targets. The pattern of errors and omissions of subject-verb agreement $(\mathrm{S}-\mathrm{V})$ and subject-predicate (S-P) was analyzed. The results showed main effects of number and gender. The data indicated that the participants produced more attraction errors of S-V when the local noun was in plural; while the most influential feature for S-P agreement was the feminine gender of the head noun of the subject. In summary, in relation to the agreement between the subject and the verb, and specifically, for the number feature, classical asymmetry was found, with greater interference when the head noun appeared in singular and the local noun in plural. The surprising fact, which could come to light given the characteristics of the design of the experiment, is that the attraction occurred more strongly when the gender of both nouns matched. With regard to agreement of subject- adjective predicate, and with respect to the gender of the nouns and the adjective, a main effect of the gender of the head noun was found, with more errors when the N1 appeared in feminine. We also found an interaction between the gender of $\mathrm{N} 1$ and the gender of N2, with more errors when the N1 appeared in feminine and N2 in masculine, and at the same time when the number of both coincided. The data suggest that it seems that the most influential feature to make the agreement between the subject and the predicate adjective is the gender of head noun, with a higher probability of interference when it appears in the feminine gender. In sum, our experimental research gathered evidence that allows us to progress in the characterization of how and when the processing of agreement and interference occurring in situations forced by experimental conditions as in natural speech. In particular, we analyze the characteristics of the gender and number features and the role of the marking concept. The data obtained in our experimental work were analyzed in terms of a general theory of processing so as to inform a production model of the still developing agreement.

Keywords: Psycholinguistics; Sentence production; Agreement; Gender; Number

\section{Introducción}

La producción del lenguaje es una actividad natural y frecuente en los seres humanos. Sin embargo, supone coordinar con precisión temporal la intervención de materiales de naturaleza diversa. Cuando un hablante produce una oración se deben transformar sus intenciones comunicativas en una secuencia de sonidos lingüísticos, para así transmitir el mensaje que desea comunicar. Esta transformación implica realizar una serie de operaciones complejas, entre ellas la operación de concordancia. Este trabajo se ocupa de investigar experimentalmente la concordancia entre el sujeto y el verbo (S-V) y entre el sujeto y el adjetivo predicativo (S-P), durante la producción de habla en tiempo real.

\section{Concordancia y errores de atracción}

Para poner en juego los rasgos que intervienen en la concordancia, las lenguas 
varían de acuerdo a cuán ricas son en la expresión superficial de estos rasgos. Por ejemplo, en español, la concordancia entre el sujeto y el verbo de la oración aplica una regla simple: los rasgos de persona y número del sustantivo núcleo del sujeto deben coincidir con los del verbo principal. Asimismo, en la concordancia entre el sujeto y el adjetivo predicativo los rasgos que deben concordar son el número y el género. Los ejemplos (1) y (2) ilustran claramente esta descripción:

Ejemplos:

(1) [La niña] SingFem es $3^{\circ}$ PersonaSing bonita SingFem

(2) [Los niños] PlMasc son $3^{\circ} \mathrm{PPl}$ lindos PlMasc

Tanto el género como el número son dos categorías morfosintácticas (RAE, 2009). El género es un rasgo inherente a los sustantivos, pero también lo adoptan los determinantes y los adjetivos que acompañan a los sustantivos a través de la concordancia y los pronombres que los sustituyen, y sirve para diferenciar el sexo del referente. El número, además de afectar a los sustantivos, adjetivos, determinantes y pronombres, también lo hace a los verbos, y opone la idea de individualidad con la de multiplicidad. En nuestra lengua, ambos rasgos tienen un sistema de valoración dual, ya que distinguen entre el singular y el plural para el número, y entre el masculino y el femenino en el caso del género; son categorías que se manifiestan por medio de desinencias o sufijos flexivos que se agregan a la raíz de los sustantivos, adjetivos, entre otros (por ejemplo, o/a-o/e-o/a en chico/chica, cliente/ clienta, león/leona para el género).

La regla descripta en los ejemplos (1) y (2) puede parecer sencilla; sin embargo, es habitual cometer errores debido a la interferencia de algún elemento. Dentro de la literatura psicolingüística, este fenómeno es conocido como atracción (Quirk, Greenbaum, Leech \& Svartvik, 1972). En los errores de atracción de número, por ejemplo, el verbo concuerda con un sustantivo local o interfiriente (en el ejemplo 3: errores) inserto en el sintagma de determinante
(SD) sujeto en lugar de concordar con el sustantivo núcleo del sujeto (estudio).

Ejemplo:

(3) *El estudio de los errores aportarán evidencia a favor de este modelo. (El * indica la agramaticalidad de la oración)

Los errores de atracción y sus características fueron profusamente utilizados en los estudios experimentales de la concordancia con el objetivo de elucidar cuáles son los dominios estructurales dentro de los cuales se realiza esta operación y cuáles son los factores que juegan un papel en el momento en el que se está procesando la concordancia, es decir, qué tipo de información toma en cuenta el sistema para producir el ajuste de los rasgos pertinentes (Bock et al., 2001; Bock \& Eberhard, 1993; Eberhard et al., 2005; Franck et al., 2008; Hartsuiker et al., 2003; Vigliocco et al., 1995, 1996, entre otros).

\section{La producción de la concordancia de número y de género}

Dentro de las investigaciones psicolingüísticas que indagan la concordancia, la mayor cantidad de trabajos experimentales se ha enfocado en el estudio de los errores de concordancia de número entre el sujeto y el verbo y éstos han sido utilizados extensamente para contribuir con datos en la discusión acerca de la modularidad o no del sistema de producción de habla (Bock \& Miller, 1991; Bock \& Levelt, 1994; Bock et al., 2001; Eberhard et al., 2005; Franck et al., 2002, 2006, 2008; Rodrigues, 2006; Vigliocco et al., 1995, 1996, 1998; Vigliocco \& Hartsuiker, 2002). Una de las características de los errores de atracción es la asimetría encontrada entre los diferentes valores del número, esto es, cuando el sustantivo núcleo del sujeto se encuentra en singular y el sustantivo interfiriente en plural, generando una concordancia plural (ver ejemplo 3). El patrón inverso, como por ejemplo "* Los estudios del error aporta evidencia...", es bastante inusual (Franck et al., 2002). Si bien, se ha mostrado que factores conceptuales, sintácticos y fonológicos tam- 
bién podrían jugar un rol en este particular patrón de errores (Antón-Méndez \& Hartsuiker, 2010; Bock \& Eberhard, 1993; Bock et al., 2001; Eberhard, 1997; Foote \& Bock, 2011; Franck et al., 2002; Franck, Vigliocco, Antón-Méndez, Collina, \& Frauenfelder, 2008; Gillespie \& Pearlmutter, 2011; Haskell \& Mac Donald, 2003; Vigliocco, et al. 1995; Vigliocco, Butterworth, \& Garrett 1996), la atracción es principalmente activada por los rasgos morfológicos involucrados (Antón-Méndez, Nicol, \& Garrett, 2002; Bock, Carreiras \& Meseguer, 2012; Bock \& Miller, 1991; Fayol, Largy, \& Lemaire, 1994; Franck et al., 2002, 2006, 2008; Hartsuiker et al., 2003; Vigliocco \& Nicol, 1998).

Si bien no son abundantes en la literatura, algunas investigaciones han reportado el fenómeno de la asimetría en otros tipos de concordancia como la de género entre el sujeto y el predicativo adjetivo (Badecker \& Kuminiak, 2007; Vigliocco \& Franck, 1999, 2001) y/o la concordancia de género y número entre el pronombre y su antecedente (Bock, Nicol, \& Cutting, 1999; Bock, Eberhard, \& Cutting, 2004). A los propósitos de este trabajo, nos detendremos en las investigaciones referentes a la concordancia sujeto-adjetivo predicativo.

Las primeras investigaciones sobre el procesamiento del género y número en concordancia sujeto-verbo y sujeto-adjetivo predicativo fueron realizadas en español (Antón-Méndez et al., 2002; Igoa \& GarcíaAlbea, 1999). En estos estudios se buscó verificar si la información de estos rasgos durante la producción de oraciones se computaba de manera independiente $\mathrm{y}$, al mismo tiempo, ver si los dos tipos de concordancia constituían un proceso único o no. En términos generales, los resultados mostraron que la concordancia de género y de número ocurre de manera independiente. Este dato es compatible con la hipótesis teórica propuesta por Picallo (2008) que sugiere que el género y el número están representados independientemente dentro del sintagma de determinante. Sin embargo, encontraron que la concordancia de número con el verbo está relacionada con la concordancia de número con el adjetivo predicativo, esto hace parecer que ambas concordancias funcionan como un proceso unitario. Además, descubrieron que los errores de género y de número son sensibles a la congruencia de otros rasgos, lo que es interpretado como el resultado de una corrección post-producción. Vigliocco y Franck (1999) también investigaron el procesamiento de la concordancia de género en estructuras predicativas del francés y del italiano. Buscaron investigar si durante la codificación de la concordancia el sistema de producción del lenguaje utiliza información conceptual referente al sexo biológico de un sustantivo y también si habría un efecto de marcación de género, similar a la asimetría encontrada para el número. Los resultados revelaron un efecto del tipo de género, con más errores de concordancia entre el sujeto y el adjetivo predicativo cuando los sustantivos no tenían asociada la información sobre el sexo del referente. De acuerdo con los autores, en los casos en los cuales el género es conceptualmente especificado, podría haber una combinación de información conceptual y morfosintáctica en el momento de la computación de los rasgos de género y esto es lo que reduciría la posibilidad de errores. La información de naturaleza conceptual, en los casos en que fuese congruente con la información gramatical, daría información redundante que serviría para el establecimiento de una correcta concordancia. Este resultado es tomado como evidencia a favor de los modelos interactivos (o no seriales) de la producción del lenguaje y de penetración de los niveles superiores por sobre los inferiores, ya que considera que el codificador gramatical usaría información disponible del nivel del mensaje para computar la concordancia (Bates \& MacWhinney, 1989; Dell, 1986; Lewis \& Vasishth, 2005; Stemberger, 1985; Vigliocco \& Hartsuiker, 2002). En relación a los efectos de marcación, los datos del estudio de Vigliocco y Franck (1999) explicitan diferencias entre las lenguas estudiadas. Para el francés se observó la asimetría 
femenino-masculino, con más errores en los preámbulos en los que el sustantivo núcleo del sujeto (N1) aparecía en femenino. Las autoras atribuyen este resultado a diferencias en la realización morfofonológica del género en esas dos lenguas: el femenino en los adjetivos en francés se hace a partir de la adición de un morfema a la forma masculina (ej. "delicat" [delicado - Masc] / "delicate" [delicado - Fem]); en cambio, en italiano (y también en español), en general, se cambia un morfema por otro (ej. "delicato" [delicado- M]/“"delicata" [delicado Fem]). Ellas explican esta diferencia aduciendo que para el francés producir erróneamente un adjetivo en la condición en que el N1 está en femenino es menos costosa (donde ocurren más errores) porque la forma es menos compleja. Otra forma de interpretar este resultado es pensando que en el francés hay una tendencia a emplear la forma no marcada del adjetivo, independientemente del género del N1.

Estudios realizados en lenguas que contienen tres valores para el género, como por ejemplo el eslovaco (Badecker \& Kuminiak, 2007), sostienen que las explicaciones brindadas, relacionadas con la subespecificación para las asimetrías de dos valores (singular-plural / masculino-femenino), no pueden aplicarse cuando existe un tercer valor en los rasgos involucrados en la concordancia. En estas lenguas parece ser que tanto el caso del sustantivo núcleo del sujeto y del sustantivo interfiriente como el género son claves activas en la memoria de trabajo que guían el procesamiento de la concordancia.

\section{Objetivos}

En el presente trabajo hemos estudiado el patrón de errores de concordancia en la producción de oraciones del español utilizando el paradigma de elicitación de errores en una tarea de completamiento de oraciones con preámbulos complejos (Bock \& Miller 1991). Se estudió la concordancia de número entre el sujeto y el verbo y la concordancia de género y número entre el sujeto y el adjetivo predicativo. Como objetivo general, en este trabajo nos propusimos investigar el procesamiento de los rasgos que entran en juego tanto en la concordancia S$\mathrm{V}$ como en la concordancia S-P en nuestra lengua. Específicamente, se intentará responder si los rasgos de género y número se procesan de la misma manera, si los rasgos tienen un valor por defecto y si los valores presentan diferencias, esto es, si el singular se diferencia del plural, y el masculino del femenino. Esto nos permitirá establecer si alguno de estos valores es más sensible al error. Además, buscamos identificar el papel del concepto de marcación.

Asimismo, en términos generales, mediante el análisis de errores de atracción de número y de género cometidos por sujetos hablantes nativos del español durante la producción de oraciones, se propuso caracterizar el mecanismo de la concordancia en el marco de un modelo de procesamiento general, teniendo en cuenta los rasgos involucrados. En este contexto, el Modelo de Recuperación de la Memoria de Trabajo (Working Memory Retrieval Model) de Badecker y Kuminiak (2007) permite explicar distintos efectos encontrados en los estudios psicolingüísticos de la producción de la concordancia de número y de género, poniéndolos en relación con medidas de procesamiento en tiempo real.

\section{Método}

\section{Participantes}

Participaron del experimento 20 sujetos hablantes nativos del español: 4 hombres y 16 mujeres. La media de edad fue de 27 años $(D S=7.86)$ y la media de escolaridad de 15.85 años $(D S=1.18)$.

\section{Materiales}

Los ítems experimentales consistían en un preámbulo complejo formado por un determinante, un sustantivo (N1 o núcleo del sujeto), un sintagma preposicional (SP) que 
incluía otro sustantivo (N2 o interfiriente). Ambos sustantivos con el rasgo semántico de [+ HUMANO]. Se utilizaron sustantivos de diferentes rangos de frecuencia de uso para que esa variable se neutralice.

Las variables manipuladas fueron las siguientes:

1) el Género de N1 y N2 (femenino-masculino), es decir, se utilizaron oraciones en las que tanto el sustantivo núcleo del sujeto como el sustantivo interfiriente aparecieron combinadamente en femenino y en masculino.

2) el Número de N1 y N2 (singular-plural), esto es, al igual que con el género, los dos sustantivos aparecían en singular y en plural.

Así, se crearon 16 condiciones divididas en 4 listas (ver ejemplos de cada condición en la Tabla 1), en un diseño de $2 \times 2 \times 2 \times 2$ (dos sustantivos: núcleo del sujeto-interfiriente; dos rasgos: género-número; dos valores para el género: femenino-masculino; y dos valores para el número: singular-plural). A cada sujeto se le administró una lista que contenía 128 targets y 42 rellenos.

\section{Procedimiento}

Se diseñó una tarea de completamiento de oraciones bajo el paradigma de elicitación de errores (Bock \& Miller, 1991). A los participantes se les presentó en forma visual la base de un adjetivo por $500 \mathrm{~ms}$. (peinad_). Luego desaparecía e inmediatamente se les presentó el preámbulo por unos 4000 $\mathrm{ms}$. Una vez que aparecía el preámbulo, los participantes debían leerlo en voz alta y luego completar la oración con el verbo ser o estar y el adjetivo flexionado. Ejemplo: El abuelo del chico está PEINADO (DmDX. Forster, K. \& Forster, J., 2003).

Los participantes fueron evaluados individualmente sentados frente a la pantalla de una computadora. Antes de los ítems experimentales, los participantes pasaron por cuatro ítems de práctica con el experimentador presente y recibieron correcciones sobre la tarea. Cada participante fue evaluado en una sesión individual de 25 minutos aproximadamente.

\section{Criterio de puntuación}

Se estudió el patrón de errores de concordancia entre el sujeto y el verbo $(\mathrm{S}-\mathrm{V})$ y entre el sujeto y el adjetivo predicativo (S-P) y el patrón de omisiones también de ambos tipos de concordancias. Por lo que las oraciones fueron puntuadas siguiendo el siguiente criterio:

1) Respuestas correctas: cuando los participantes leían correctamente el preámbulo y lo completaban utilizando la forma correcta del verbo y/o del adjetivo. Ej.: El cuñado de los verduleros está peinado.

2) Errores de concordancia S-V: el verbo era producido con una marca de número incorrecta. Ej.: El cuñado de los verduleros están peinados.

3) Errores de concordancia S-P: el adjetivo era producido con una marca de género y/o número incorrecta. Ej.:*El cuñado de las verduleras están peinadas.

4) Omisiones $S-V$ : cuando el verbo no era producido. Ej.: El cuñado de los verduleros...

5) Omisiones S-P: cuando el adjetivo no era producido. Ej.: El cuñado de los verduleros está...

\section{Análisis de los datos}

Se llevaron a cabo análisis de varianza por ítem y por sujeto como factores aleatorios y como medida dependiente la transformación de la proporción de los errores de concordancia y las respuestas omitidas (Jaeger, 2008). Las variables manipuladas (género y número de $\mathrm{N} 1$, y género y número de N2) fueron parte de un diseño intra-sujeto e intra-ítem.

\section{Resultados}

\section{Análisis de la concordancia sujeto-verbo}

El análisis de las respuestas en la concordancia $\mathrm{S}-\mathrm{V}$ arrojó que de un total de 2560 respuestas, 2414 fueron correctas (94.29\%); 33 fueron errores de concordan- 
cia sujeto-verbo (1.3\%) y 113 fueron omisiones $(4.41 \%)$.

\section{Errores de concordancia S-V}

La Tabla 2 muestra las medias y las desviaciones típicas de los errores de concordancia sujeto-verbo para cada condición experimental. Se produjeron errores en casi todos los pares de sustantivos, por lo que la frecuencia de uso de los ítems léxicos quedó neutralizada y no parece ser un factor influyente para la generación de errores.

Los ANOVAs por ítem y por sujeto mostraron un efecto principal del Número de N2 $\left[\mathrm{F} 1_{(1,19)}=22.92, p=.000 ; \mathrm{F} 2(1,19)=6.59, p=\right.$ $.019]$, con más errores producidos por los participantes cuando el N2 estaba en plural. El análisis por ítem mostró una interacción entre el Número de N1 y Número de N2 $\left[\mathrm{F}{ }_{(1,19)}=6.66, p=.010\right]$ con más errores cuando el N1estaba en singular y el N2 en plural. También se encontró una interacción entre los cuatro factores (género y número del $\mathrm{N} 1$, y género y número del $\mathrm{N} 2$ $\left[\mathrm{F} 1_{(1,19)}=7.17, p=.007 ; \mathrm{F} 2(1,19)=4.63, p=\right.$ $.044]$, con mayor cantidad de errores producidos cuando el N1 y el N2 estaban en singular-plural respectivamente y cuando coincidía el género de ambos sustantivos (no hay diferencias significativas entre las condiciones en las que aparecen los dos sustantivos en femenino o los dos en masculino. Ej.*El cuñado de los verduleros están peinados) (Ver Figura 1).

\section{Omisiones S-V}

La Tabla 2 muestra las medias y las desviaciones típicas para de las omisiones de la concordancia sujeto-verbo para cada condición experimental. El análisis de varianza por ítem también mostró un efecto principal de número de $\mathrm{N} 2\left[\mathrm{~F}_{(1,19)}=10.53, p=.001\right]$ con más respuestas omitidas cuando el sustantivo interfiriente aparecía en plural (Ej. El cuñado de los verduleros...). La interacción encontrada involucraba los factores Número de N1 y Género de N1 y N2 [F1 1 , $19)=7.46, p=.006]$.

\section{Análisis de la concordancia sujeto-adje- tivo predicativo}

El análisis de las respuestas en la concordancia S-P arrojó que de un total de 2560 respuestas, 2254 fueron correctas (88.04\%); 128 fueron errores de concordancia sujetopredicativo $(5 \%)$ y 178 fueron omisiones (6.95\%).

\section{Errores de concordancia S-P}

La Tabla 3 muestra las medias y las desviaciones típicas de los errores de la concordancia sujeto-adjetivo predicativo para cada condición experimental. El análisis de varianza por ítem mostró un efecto principal del género de $\mathrm{N} 1\left[\mathrm{~F} 1_{(1,19)}=3.91, p=\right.$ .048], con más errores cuando el sustantivo núcleo del sujeto aparecía en género femenino (Ver Figura 2).

Los ANOVAs por ítem y por sujeto mostraron una interacción entre el género de $\mathrm{N} 1$ y el género de $\mathrm{N} 2\left[\mathrm{~F} 1_{(1,19)}=39.68, \mathrm{p}=\right.$ $.000 ; \mathrm{F} 2(1,19)=35.80, p=.000]$, con más errores producidos por los participantes cuando el género de ambos sustantivos estaba en desacuerdo (Ej.: * La cuñada del verdulero está peinado).

También se encontró una interacción entre los cuatro factores (género y número del N1, y género y número del N2 $\left[\mathrm{F} 1_{(1,19)}=19.78\right.$, $p=.000 ; \mathrm{F} 2(1,19)=17.36, p=.001]$. La mayor cantidad de errores fueron de género (ver Figura 3 ) y en las condiciones en las que el género estaba en desacuerdo y el rasgo de número coincidía (el análisis post hoc no mostró diferencias significativas entre las condi- ciones N1SingMasc, N2SingFe y N1PlFem N2P1Masc) .

\section{Omisiones S-V}

La Tabla 3 muestra las medias y las desviaciones típicas para cada condición experimental para las omisiones de la concordancia S-P. El análisis de varianza por ítem y por sujeto mostró un efecto principal del género de $\mathrm{N} 1\left[\mathrm{~F} 1_{(1,19)}=6.01, p=.014 ; \mathrm{F} 2_{(1,19)}=\right.$ $6.64, p=.018]$ con más respuestas omitidas 
cuando el sustantivo núcleo del sujeto aparecía en femenino.

En resumen, en relación con la concordancia entre el sujeto y el verbo, y específicamente, para el rasgo de número, se encontró la asimetría clásica, con mayor interferencia cuando el sustantivo núcleo aparecía en singular y el interfiriente en plural. El dato sorprendente, que pudo salir a la luz dada las características del diseño del experimento, es que la atracción se dio más fuertemente cuando el género de ambos sustantivos coincidía. Con respecto a la concordancia sujeto-adjetivo predicativo, y en lo que respecta al género de los sustantivos y del adjetivo, se encontró un efecto principal del género del sustantivo núcleo del sujeto, con más errores cuando el N1 aparecía en femenino. También se encontró una interacción entre el género del N1 y el género del N2, con más errores cuando el N1 aparecía en femenino y el N2 en masculino $\mathrm{y}$, al mismo tiempo, cuando el número de ambos coincidía. Los datos sugieren que parece ser que el rasgo más influyente para realizar la concordancia entre el sujeto y el adjetivo predicativo es el género del sustantivo núcleo, con mayor probabilidad de interferencia cuando éste aparece en género femenino.

\section{Discusión}

En el presente experimento se ha estudiado el patrón de errores y de omisiones de concordancia en la producción de oraciones del español utilizando el paradigma de elicitación de errores (Bock \& Miller, 1991). Se diseñó una tarea de completamiento de oraciones con preámbulos en los que se manipuló el género y el número del sustantivo núcleo del sujeto, y el género y el número de un sustantivo interfiriente o local que estaba dentro de un sintagma preposicional. Así, se estudió la concordancia de número entre el sujeto principal de la oración y el verbo, y la concordancia de género y número entre el sustantivo principal y el adjetivo predicativo.
Los datos del presente experimento evidencian que los distintos rasgos involucrados durante el procesamiento de la concordancia en español se procesan de manera diferente y dependiente. Paralelamente, cada rasgo asume un valor por defecto. En este sentido la marcación influye en el procesamiento de la concordancia, así tanto el género femenino como el número plural, rasgos marcados en el español, interfieren de distinta manera que su valor opuesto: masculino y singular, respectivamente.

En relación a los efectos de marcación nuestros datos evidencian una asimetría en el rasgo de número en la concordancia entre el sujeto y el verbo: se produjeron más errores cuando el sustantivo núcleo del sujeto (N1) aparecía en singular y el sustantivo interfiriente (N2) en plural. Un dato curioso es que esta asimetría apareció fuertemente asociada a la coincidencia de género. Esto es, la mayoría de los errores de número en el verbo se evidenciaron cuando tanto el sustantivo núcleo del sujeto como el interfiriente presentaban el mismo género (Ej. *El cuñado de los verduleros están peinados o * La cuñada de las verduleras están peinadas). Numerosas investigaciones estudiaron la marcación de número y mostraron, en términos generales, un incremento de errores de concordancia cuando los sustantivos núcleos del sujeto no marcan número (Bock et al., 2001; Eberhard, 1997; Franck, Vigliocco, Antón-Méndez, Collina \& Frauenfelder, 2008; Hartsuiker, Schriefers, Bock \& Kikstra, 2003; Vigliocco et al., 1995). Generalmente, se asume que el rasgo del sustantivo interfiriente (plural) es copiado a la proyección más alta del sintagma de determinante y allí entra en competencia con el rasgo asignado al sustantivo núcleo del sujeto (Kempen \& Hoenkamp, 1987). También la asimetría de número puede explicarse destacando que para los sustantivos plurales hay una especificación de plural subyacente, mientras que para los sustantivos singulares no existe tal especificación de número (Eberhard, 1997). Otra explicación toma en cuenta los parámetros de uso, como la frecuencia de activación de 
un rasgo (Dell, 1986). En enfoques como este último, los rasgos no marcados se activarían por defecto, mientras que la utilización de un rasgo marcado supondría o bien costo adicional, o bien mayor propensión a cometer errores (Badecker \& Kuminiak, 2007; Lorimor et al., 2008). De este modo, la marcación parece tener una influencia importante en el procesamiento y una incidencia significativa en la producción de errores.

Si bien la asimetría de número encontrada, ya ha sido explicada desde diferentes modelos de producción de la concordancia (Eberhard, 2005; Franck et al., 2002; Vigliocco et al., 1995), la mayor incidencia de errores de número cuando hubo coincidencia en el rasgo de género se puede interpretar a la luz de un modelo que tome en cuenta aspectos como la semejanza entre los ítems activos para realizar el procesamiento de la concordancia. En este sentido, el Modelo de recuperación de la memoria de trabajo establece que la interferencia de un distractor durante la recuperación fuertemente relacionada con la similaridad al blanco (Badecker \& Kuminiak, 2007; Lewis \& Vasishth, 2005). Así, en lo que respecta a los resultados, la similaridad del rasgo de género, hace que el sustantivo núcleo del sujeto y el sustantivo interfiriente sean más semejantes y esto mejora la atracción de número (se retomará esta explicación en los siguientes párrafos).

En cuanto al género, los datos sugieren un fuerte efecto de marcación de este rasgo en el análisis del procesamiento de la concordancia sujeto-adjetivo predicativo. La dirección de los errores muestra que los participantes produjeron más errores cuando el N1 aparecía en femenino y cuando había un desacuerdo en los rasgos de género de N1 y el N2 (Ej.: *La cuñada del verdulero está peinado). En algunos de los experimentos ya descriptos anteriormente se observó también una asimetría en la marcación en francés y español, pero no en italiano (Vigliocco \& Franck, 1999; AntónMendéz et al., 2002). Esta asimetría va en dirección contraria a la reportada para el número, ya que aparecen más errores cuando el sustantivo núcleo está marcado (femenino). Antón-Méndez et al. (2002) explicaron esta diferencia haciendo hincapié en el concepto de marcación. Según los autores, mientras que el número default (singular) sería no-marcado, el género default (masculino) no parece ser no-marcado. En este sentido, en los casos en los que se observa un efecto de género no sería una cuestión de asimetría asociada a la marcación, sino una tendencia de los hablantes a emplear la forma masculina cada vez que tienen una duda. Es decir, el sistema de monitoreo es menos sensible a este tipo de error y no bloquea la producción de la forma masculina. Los datos del presente experimento parecen apoyar esta idea y están en consonancia con los reportados en otros trabajos (Sánchez, Sevilla \& Bachrach, 2016; Badecker \& Kuminiak, 2007). Sin embargo, un dato importante complementa esta hipótesis permitiendo nuevas explicaciones. Al mismo tiempo, se produjeron más errores de género cuando el número de ambos sustantivos también coincidía. Aquí también, tal como fue explicitado para el rasgo de número, la explicación desde un modelo de procesamiento como el Modelo de recuperación de la memoria de trabajo (Badecker \& Kuminiak, 2007) abre un camino fructífero. En el marco de este modelo se asume que una vez que se ha producido o recuperado un sintagma nominal -o más generalmente, algún ítem marcado con rasgos phi-, éste se mantiene activo en la memoria de trabajo (MT) -o al menos sus rasgos asociados. Luego, una vez que un ítem que requiere la selección de una forma de concordancia morfológica (por ejemplo, un verbo o un adjetivo) se recupera para la producción, se comienza una búsqueda en la MT de un antecedente que sea relevante (el controlador). En este sentido, el procesador, presionado por el tiempo y por las restricciones de la memoria de trabajo (Lewis, Vasishth \& Van Dyke, 2006), necesita una clara indicación de la naturaleza de los elementos que unirá por medio de la operación de concordancia. Lo rasgos morfológicos, 
entre otros, funcionan como claves en la recuperación y permiten elegir el sustantivo correcto para ejecutar el cómputo de manera exitosa. El efecto de género gramatical explicado desde un modelo de recuperación de claves puede coexistir con otros mecanismos que afectan la concordancia, como la asimetría de número o los efectos semánticos. Los resultados de este experimento también parecen evidenciar que tanto el número gramatical como el género gramatical del sujeto se mantienen en la memoria de trabajo y que el rasgo de género se puede utilizar para ayudar a localizar correctamente el rasgo de número del N1 durante la recuperación. Estos datos suman evidencia junto con los datos de marcación de número de la concordancia sujeto-verbo para un modelo de producción de la concordancia que se enfoca directamente en el procesamiento.

En los datos que se reportaron en este artículo parece que los rasgos de los sustantivos involucrados en el sujeto de la oración son factores que funcionan como claves y provocan interferencia. Cuando aparece un conflicto, producto de las variables manipuladas, que induce la presencia de un posible interventor activo, el sistema de producción se vuelve más proclive al error. Se trata de una situación en la cual el costo de procesamiento es alto, ya que se mantienen en la memoria de trabajo dos potenciales relaciones de concordancia con dos elementos distintos (sustantivo núcleo y sustantivo interfiriente) que ocupan dos posiciones estructurales activas para la concordancia. La mayor ventaja de considerar la atracción en el marco de un modelo de MT es que el procesamiento gramatical es implementado sobre la búsqueda de un controlador de la concordancia en una estructura abstracta posiblemente motivada por un mecanismo más general, como puede ser la similaridad. La información gramatical funciona sólo como un conjunto de claves que impulsan la búsqueda.

En suma, la presente investigación experimental recogió evidencia que permite avanzar en la caracterización de cómo y cuándo se produce el procesamiento de la concordancia y las interferencias que se observan tanto en situaciones forzadas por las condiciones experimentales como en el habla natural. En particular, se analizan las características de los rasgos de género y número y el rol del concepto de marcación. Los datos obtenidos en este trabajo experimental fueron analizados en términos de una teoría general del procesamiento de modo que permiten informar un modelo de producción de la concordancia aún en desarrollo. 
Tabla 1

Ejemplos de preámbulos experimentales

\begin{tabular}{|c|c|c|}
\hline Condkith & Adjetiws & Proimbulo \\
\hline 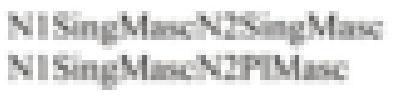 & \multirow[t]{15}{*}{ pinad } & $\begin{array}{l}\text { Dl atudo dal chiso } \\
\text { El abuclo de kx chicos }\end{array}$ \\
\hline NISingMare N26 ingFtm & & B1 abuclo de L cha \\
\hline NISingMise W2PFFem & & El abucto de lis chess \\
\hline NISinpFemv2singslme & & Lubelu del atw \\
\hline NISingFemNzPIMare & & Labula de bo chow \\
\hline NISingFemW2singFut & & Lubata de billo \\
\hline NISingFemNzPIFem & & Lubsh of lo ditom \\
\hline NIPMuN2SinpMase & & Las abukiri del chico \\
\hline NIFMneNaPMn & & Lew abuelen de lon chicos \\
\hline MHFMw2singFem & & Low abuelow de la chika \\
\hline NIFMneNaPHF" & & La abuelow de las chicas \\
\hline WIFFemw23ingMire & & Las abuclas del chico \\
\hline NiflemwarMuk & & Les abuclas de los chlow \\
\hline NIFFemw2singFem & & Les abuclas de la chica \\
\hline NIFFemvarFem & & Las abuelas de las chican \\
\hline
\end{tabular}

Nota:

N1: Sustantivo núcleo del sujeto

N2: Sustantivo interfiriente

Sing: Número Singular

$\mathrm{Pl}$ : Número Plural

Masc: Género Masculino

Fem: Género Femenino 
Tabla 2

Media y deviación estándar por condición experimental de acierto-error y acierto-omisión de la concordancia $S$ - $V$

\begin{tabular}{|c|c|c|c|c|}
\hline \multirow[b]{2}{*}{ Candician } & \multicolumn{2}{|c|}{ Enotes Sy } & \multicolumn{2}{|c|}{ Dmiswots S- } \\
\hline & M & $D F$ & M & $D T$ \\
\hline MISinpMaseN2SinpMane & $7,+60$ & 00 & 7,07 & $2 \times 9$ \\
\hline NISinpMas N2FMase & B. & 337 & 6.3 & 4.16 \\
\hline NISinpMus WasingFem & 7.60 & 00 & 7.22 & 238 \\
\hline NISinpMasckafFem & 7.50 & 122 & 6.93 & 3.11 \\
\hline NISinpFemw2singMire & 7.60 & an & 7.03 & 239 \\
\hline NISinpFemv2PIMas & 7.10 & 270 & 6.5 & 3.55 \\
\hline NISinpFemvizhefem & 7.60 & 00 & 64 & 401 \\
\hline NISingFemviPIFem & 690 & 1.IB & 6.27 & 430 \\
\hline NIPAMakNisingMw & 7.60 & 00 & 7.31 & 204 \\
\hline NIPHMakN2PMML & 7.49 & 123 & 6.6 & 369 \\
\hline NIPAMwN2SinpFem & 7.50 & 122 & 6.4. & 332 \\
\hline NIPAManIPIF"n & 7.39 & 1.74 & 6.5 & 3.85 \\
\hline NIPIFo-N2Ging Mase & 7.40 & 122 & 6.93 & 3.11 \\
\hline NIPAFe= N2PHMIK & 69s & 300 & 5.69 & 4.31 \\
\hline NIPIFem NaSingFem & 7.40 & 122 & 6.84 & 332 \\
\hline NIPIFem N2P1Fem & 7.60 & 00 & 6.A & 332 \\
\hline
\end{tabular}

Nota:

M: Media / DS: Desviación estándar

N1: Sustantivo núcleo del sujeto / N2: Sustantivo interfiriente

Sing: Número Singular / Pl: Número Plural

Masc: Género Masculino / Fem: Género Femenino 
Tabla 3

Media y deviación típica por condición experimental de acierto-error y acierto-omisión de la concordancia $S-P$

\begin{tabular}{|c|c|c|c|c|}
\hline \multirow[b]{2}{*}{ Condición } & \multicolumn{2}{|c|}{ Emones S-P } & \multicolumn{2}{|c|}{ Ontsiones 8-P } \\
\hline & $M$ & $D T$ & 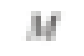 & $D T$ \\
\hline 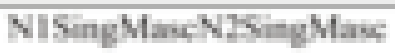 & $7+4$ & 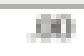 & 691 & 1III \\
\hline NISingMine VzPMase & $6 \%$ & 2.97 & 571 & 357 \\
\hline NISingMira N23i-gFem & 5.92 & 4.77 & 64 & 305 \\
\hline NISinpMire N2PIFem & b.64 & 363 & s.49 & 34 \\
\hline NISingFemN2SingMlus & 6.34 & 4.13 & 657 & 362 \\
\hline NISinģFemk2PIMars & b.6 & 3.61 & 66 & 361 \\
\hline NiSingFemN2singFont & 7.40 & 00 & 5.98 & 469 \\
\hline NISingFemN2PIFem & b.M & 322 & 550 & 343 \\
\hline NHPMak2singMase & 7.40 & 1.72 & 7.11 & 266 \\
\hline NIPMnkNPMak & 7.14 & 2.47 & 643 & 406 \\
\hline WIFMuk2GingFem & 7.19 & 2.46 & 652 & 3.40 \\
\hline NIFMaNaPAFem & 6.35 & 4.17 & s.ts & 431 \\
\hline NIFFamv26npMire & 6.4 & 4.16 & 63 & 4.16 \\
\hline NHFFemvaPMa & 3.15 & 3.60 & 5.71 & 302 \\
\hline NIFFemvishngFem & 6.67 & 3.49 & 639 & 4.12 \\
\hline NIFFemW2FFem & 7.44 & 1.78 & s0 & 460 \\
\hline
\end{tabular}

Nota:

M: Media / DS: Desviación típica

N1: Sustantivo núcleo del sujeto / N2: Sustantivo interfiriente Sing: Número Singular / Pl: Número Plural

Masc: Género Masculino / Fem: Género Femenino 


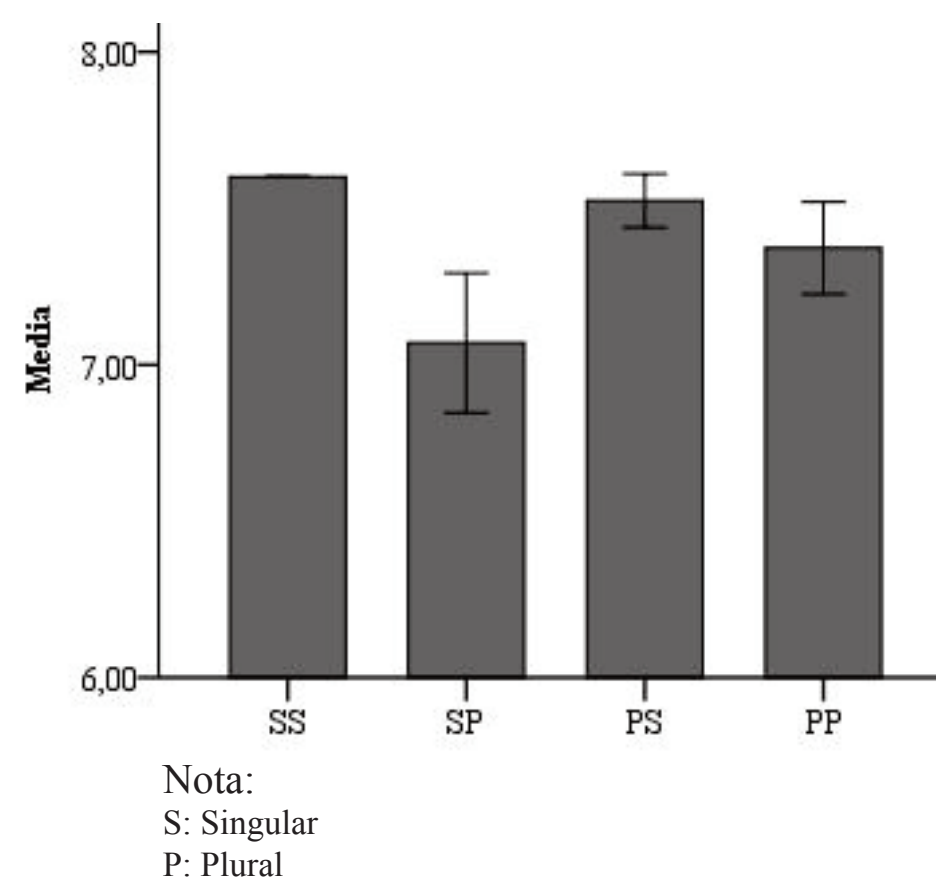

Figura 1. Media de aciertos entre el sujeto y el verbo en función del Número de N1 y el Número de N2

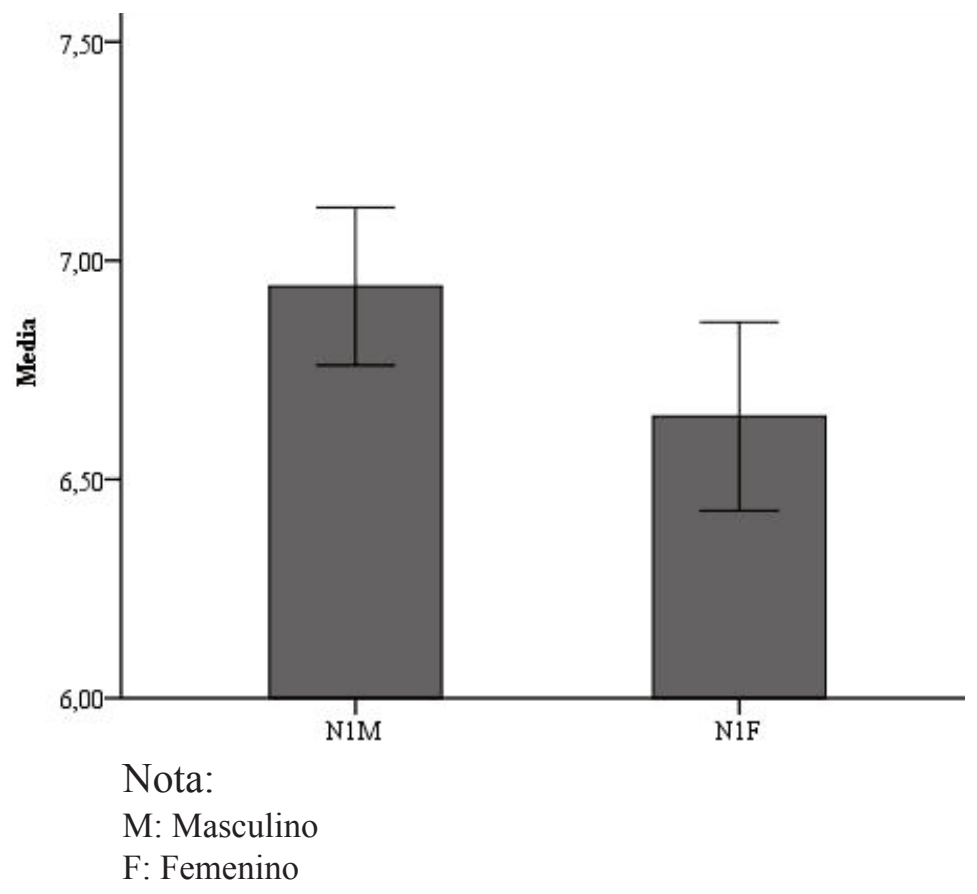

Figura 2. Media de aciertos entre el sujeto y el adjetivo predicativo en función del Género de N1 


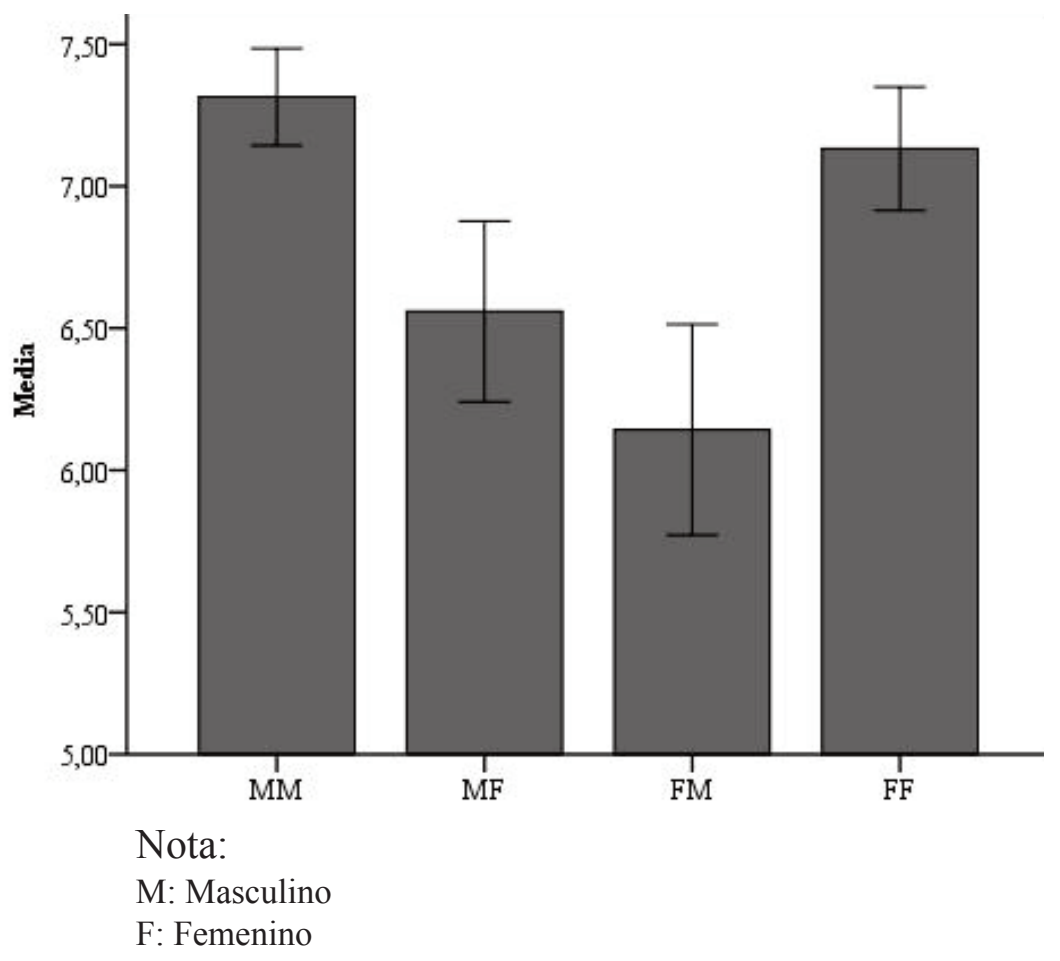

Figura 3. Media de aciertos entre el sujeto y el adjetivo predicativo en función de la interacción entre el Género de N1 y el Género de N2

\section{Referencias bibliográficas}

Antón-Méndez, I., \& Hartsuiker, R.J. (2010). Morphophonological and conceptual effects on Dutch subject-verb agreement. Language and Cognitive Processes, 25, 728-748. http://dx. doi.org/10.1080/01690960903513818

Antón-Méndez, I., Nicol, J. \& Garrett, M.F. (2002). The relation between gender and number agreement processing. Syntax, 5(1), 1-25.. http://dx.doi.org/10.1111/1467-9612. 00045

Badecker, W. \& Kuminiak, F. (2007). Morpho logy, agreement and working memory retrieval in sentence production: Evidence from gender and case in Slovak. Journal of Memory and Language, 56, 65-85. http: //dx.doi.org/10.1016/j.jml.2006.08.004

Bates, E., \& MacWhinney, B. (1989). Functionalism and the Competition Model. En B. MacWhinney, \& E. Bates (Ed.), The cross linguistic study of sentence processing. New York: Cambridge University Press.

Bock, K. \& Eberhard, K.M. (1993). Meaning, sound and syntax in English number agreement. Language and Cognitive Processes, 8, 57-99. http://dx.doi.org/10.1080/01690969308 406949

Bock, K. \& Levelt, W.J.M. (1994). Language production: grammatical encoding. En M. Gernsbacher (comp.), Handbook of Psycholinguistics. New York: Academic Press.

Bock, K. \& Miller, C. (1991). Broken Agreement. Cognitive Psychology, 23, 45-93. http://dx.doi. org/10.1016/0010-0285(91)90003-7

Bock, K., Carreiras, M., \& Meseguer, E. (2012). Number Meaning and Number Grammar in English and Spanish. Journal of Memory and Language, 66, 17-37. http://dx.doi.org/10.1016/ j.jml.2011.07.008. 
Bock, K., Eberhard, K.M. \& Cutting, J.C. (2004). Producing number agreement: how pronouns equal verbs. Journal of Memory and Language, 51(2), 251-278. https://doi. org/10.1016/j.jml.2004.04.005

Bock, K., Eberhard, K.M., Cutting, J.C., Meyer, A. \& Schriefers, H. (2001). Some attractions of verb agreement. Cognitive Psychology, 43, 83-128. http://dx.doi.org/10. 1006/cogp.2001.0753

Bock, K., Nicol, J., \& Cutting, J.C. (1999). The ties that bind: Creating number agreement in speech. Journal of Memory and Language, 40, 330-346. https://doi.org/10. 1006/jmla.1998.2616

Dell, G.S. (1986). A spreading activation model of retrieval in sentence production. Psychological Review, 93, 283-321. http: //dx.doi.org/10.1037/0033-295X.93.3.283

Eberhard, K.M. (1997). The marked effect of number on subject-verb agreement. Journal of Memory and Language, 36, 147-164. http://dx.doi.org/10.1006/jmla.1996.2484

Eberhard, K.M., Cutting, J.C., \& Bock, J.K. (2005). Making syntax of sense: Number agreement in sentence production. Psychological Review, 112(3), 531-559. http://dx. doi.org/10.1037/0033-295X.112.3.531

Fayol, M., Largy, P. \&Lemaire, P. (1994).When cognitive overload enhances subject-verb agreement errors: a study in French written language. Quarterly Journal of Experimental Psychology, 47, 437-464. http://dx.doi. org/10.1023/A:1008038 127807

Foote, R. \& Bock, J. K. (2011). The role of morphology in subject-verb number agreement: A comparison of Mexican and Dominican Spanish. Language \& Cognitive Processes, 27(3), 429-461. http://dx.doi.org /10.1080/01690965.2010.550166

Forster, K.I. \& Forster, J.C. (2003). DMDX: A windows display program with millisecond accuracy. Behavior Research Methods, Instruments \& Computers, 35(1), 116-124. http://dx.doi.org/10.3758/BF03195503
Franck, J., Lassi, G., Frauenfelder, U.H. \& Rizzi, L. (2006). Agreement and Movement: A syntactic analysis of attraction. Cognition, 101, 173-215. http://dx.doi.org/10.1016/j. cognition.2005.10.003

Franck, J., Vigliocco, G., \& Nicol, J.L. (2002). Subject-verb agreement errors in French and English: The role of syntactic hierarchy. Journal of Language and Cognitive Processes, 17(4), 371-404. http://dx.doi.org/10.10 $80 / 01690960$

Franck, J., Vigliocco, G., Antón-Méndez, I., Collina, S. \& Frauenfelder, U.H. (2008). The interplay of syntax and form in sentence production: A cross-linguistic study of form effects on agreement. Language and Cognitive Processes, 23(3), 329-374. http://dx. doi.org/10.1080/01690960701467993

Gillespie, M. \& Pearlmutter, N.J. (2011). Hierarchy and scope of planning in subject-verb agreement production. Cognition, 118, 377397. http://dx.doi.org/10.1016/j.cognition. 2010.10.008

Hartsuiker, R.J., Schriefers, H.J., Bock, J.K. \& Kikstra, G. M. (2003). Morphophonological influences on the construction of subjectverb agreement. Memory \& Cognition, 31, 1316-1326. http://dx.doi.org/10.3758 /BF03 195814

Haskell, T.R. \& MacDonald, M.C. (2003). Conflicting cues and competition in subject-verb agreement. Journal of Memory and Language, 48, 760-778. http://dx.doi .org/10.10 16/S0749-596X(03)00010-X

Igoa, J.M. y García-Albea, J.E. (1999). Unidades de planificación y niveles de procesamiento en la producción del lenguaje. En M. De Vega y F. Cuetos (Coord.), Psicolingüística del español (Pp. 380-384; 389-428; 441-456). Madrid: Trotta.

Jaeger, T.F. (2008). Categorical Data Analysis: Away from ANOVAs (transformation or not) and towards Logit Mixed Models. Journal of Memory and Language, 59, 434-446. http:// dx.doi.org/10.1016/j. jml.2007. 11.007 
Kempen, G. \& Hoenkamp, E. (1987). An incremental procedural grammar for sentence formulation. Cognitive Science, 11, 201258. http://dx.doi.org/10.1207/s15516709 cog1102_5

Lewis, R.L. \& Vasishth, S. (2005). An activation-based model of sentence processing as skilled memory retrieval. Cognitive Science, 29, 1-45. http://dx.doi.org/10.1207/s15516 709cog0000_25

Lewis, R.L., Vasishth, S., \& Van Dyke, J.A. (2006). Computational principles of working memory in sentence comprehension. Trends in Cognitive Science, 10(10), 447454. http://dx.doi.org/10.1016/j.tics. 2006. 08.007

Lorimor, H., Bock, J.K., Zalkind, E., Sheyman, A., \& Beard, R. (2008). Agreement and attraction in Russian. Language and Cognitive Processes, 23, 769-799. http://dx.doi.org /10.1080/01690960701774182

Picallo, M.C. (2008). Gender and Number in Romance. Lingue e Linguaggio, VII(1), 4766.

Quirk, R., Greenbaum, S., Leech, G., \& Svartvik, J. (1972). A grammar of contemporary English. London: Longman.

Real Academia Española (2009). Nueva gramática de la lengua española. Manual. Madrid: Espasa Libros.

Rodrigues, E. dos S. (2006). Processamento da concordância de número entre sujeito e verbo na produção de sentenças. Tese de Doutorado, Depto. de Letras, PUC-Rio.

Sánchez, M.E., Sevilla, Y. \& Bachrach, A. (2016). Agreement processing in control and raising structures. Evidence from sentence production in Spanish. Lingua 177, 60-77. http://dx.doi.org/10.1016/j.lingua.2015.12. 014

Stemberger, J.P. (1985). An interactive activation model of language production. En A. Ellis (Ed.). Progress in the psychology of language (Vol. I, pp. 143-186). London: Erlbaum.

Vigliocco, G. \& Franck, F. (2001). When sex affects syntax: Contextual influences in sentence production. Journal of Memory and Language 45, 368-390. http://dx.doi.org/ 10.1006/jmla.2000.2774

Vigliocco, G. \& Franck, J. (1999). When sex and syntax go hand in hand: Gender agreement in language production. Journal of Memory and Language, 40, 455-478. http: //dx.doi.org/10.1006/jmla.1998.2624

Vigliocco, G. \& Hartsuiker, R. (2002).The interplay of meaning, sound and syntax in sentence production. Psychological Bulletin, 128(3), 442-472. http://dx.doi.org/10.1037 //0033-2909.128.3.442

Vigliocco, G., \& Nicol, J. (1998). Separating hierarchical relations and word order in language production. Is proximity concord syntactic or linear? Cognition, 68, 13-29. http://dx.doi.org/10.1016/S0010-0277 (98)00041-9

Vigliocco, G., Butterworth, B. \& Garrett, M.F. (1996). Subject-verb agreement in Spanish and English: Differences in the role of conceptual constraints. Cognition, 61, 261-298.

Vigliocco, G., Butterworth, B., \& Semenza, C. (1995) Constructing Subject-Verb Agreement in Speech: The Role of Semantic and Morphological Factors. Journal of Memory and Language, 34, 186-215. 
